\title{
Plantar pressure variation during jogging with different heel height
}

\author{
Y.D. Gu ${ }^{\mathrm{a}, *}$, D. Sun ${ }^{\mathrm{a}}$, J.S. Li ${ }^{\mathrm{a}}$, M.R. Graham ${ }^{\mathrm{b}}$ and X.J. Ren ${ }^{\mathrm{c}}$ \\ ${ }^{\mathrm{a}}$ Faculty of Sport Science, Ningbo University, Zhejiang, P.R. China \\ ${ }^{\mathrm{b}}$ Institute of Health, Medical Science and Society Science, Glyndwr University, Wrexham, Wales, UK \\ ${ }^{\mathrm{c}}$ School of Engineering, Liverpool John Moores University, Liverpool, UK
}

\begin{abstract}
This paper presents the key testing and analysis results of an investigation on the effect of heel height on the plantar pressure over different foot areas in jogging. It is important in improving the understanding of jogging with high heels and damage/injury prevention. It can also potentially guide the development of suitable/adaptive exercise schemes in between daily activities with high heels. In this work, plantar pressure data were collected from 10 habituated healthy female subjects (aged 21-25 years) at their natural jogging speed with three different conditions: flat heeled shoes $(0.8 \mathrm{~cm})$, low heeled shoes $(4.0 \mathrm{~cm})$, and high heeled shoes $(6.6 \mathrm{~cm})$. Data analysis showed significantly differences in plantar pressure distribution associated with the heel heights with increased pressure in the first metatarsal region and decreased pressure in the lateral metatarsal and midfoot sections. However, there is no significant alteration of plantar pressure in the central area of the forefoot with jogging gait.
\end{abstract}

Keywords: Plantar pressure, jogging, high-heel shoes

\section{Introduction}

According to surveys on footwear, between $39 \%$ and $69 \%$ of women wear high heels on a daily basis $[1,2]$. One of the most important benefits for women wearing high-heeled shoes is that it increases the femininity of gait, shoes with heels make their wearer appear to be taller and to have longer and thinner legs and more gracious posture. Women's pursuit and enthusiasm for high heels has been enduring despite the fact that the intensity of their daily movement has been greatly increased in a modern life. In addition to intrinsic safety issues associate with the difficulty in balancing and movement, wearing high heels could also have detrimental effects on gait and lower limbs function. The possible negative effects of high-heeled shoes on

${ }^{*}$ Corresponding author: Y.D. Gu, Faculty of Sport Science, Ningbo University, Zhejiang 315211, P.R. China. Tel.: +86 574 87600456; Fax: +86 574 87600456; E-mail: guyaodong@ hotmail. com. hallux valgus [3-6], the development of degenerative joint disease [7-10] have been reported.

The issue on high heeled walking has been researched in several biomechanical studies. It is suggested that walking with high heels leads to changes in load distribution beneath the feet, increase of the foot bone internal stress $[11,12]$, decrease of the contact area and transfer of the center of pressure from midfoot to forefoot [12-14]. Another distinct difference reported is that peak pressure with positive correlation to the heel height shifting from the lateral forefoot to medial forefoot: from the area of 3rd, 4th and 5th metatarsal head to 1 st and 2nd metatarsal head [11, 13, $15,16]$. These previous findings could be correlated to high-heel wearing induced foot pain and uncomfortableness. In a recent work, Gu et al. [17] investigated the effect of high heels on plantar pressure on the shoeground surface and found that peak pressure increased not only in the fore part of sole but also the heel region. Most of these works were based slow space walking, while issues on potential effects of high heel on high 
intensity movement such as jogging is lees known. Running is potentially more injurious due to the striking impact of the foot being transferred up the lower kinetic chain at a higher rate [18]. Jogging could be a little bit safer comparing to running, as maximum force and peak pressure were lower $[19,20]$. However, slow jogging (between 1.5 and $3 \mathrm{~m} / \mathrm{s}$ ) produces vertical GRF up to 1.6 times more than walking at the same speed, thus making jogging a potentially more injurious activity as compared to walking [21]. This may have directly influences on high heel wearers and the understanding of such a process is becoming increasingly important with increasing space of modern society. Wearing high heeled shoes seems to be a currently global trend, because many international companies believed that female workers should wear high heels in order to show courtesy [28]. What's more, some companies have stipulated in the agreement that female workers should wear high heels in order to maintain professional images [22]. In these cases, wearing high heels have to be done out of necessity as part of a job; in fact, running matches in high heels was interestingly being worldwide. More detailed understanding the foot mechanics for this situation is required for improving the safety and comfortableness. However, there was very few literature specifically related to high heels in relatively high-intensity movement rather than walking. It is very important to discover plantar pressure pattern changes under different heel heights condition while jogging at a natural speed. The primary purpose of this study is to establish the characteristics of plantar pressure during jogging with increased heel height. The changes in loading and subject adaptability may be amplified in jogging comparing to walking. Detailed study of the loading pattern will provide some technical directions for optimizing design attributes of high heel shoes particularly in improving the safety factors.

\section{Materials and methods}

\subsection{Participants}

Ten young healthy females, aged 21-25 years $(23.88 \pm 1.65$; mean \pm SD) with no previous/current foot injury, participated in this study voluntarily Subjects with any pathologies that could have affected the research and its results were not qualified for participation. The average height and weight of the participants was $162.00 \pm 3.30 \mathrm{~cm}$ and $52.19 \pm 3.68 \mathrm{~kg}$, respectively. All those tested had been wearing highheeled shoes more than two years (including two years) with a frequency of about 4 times a week.

\subsection{Instruments}

Jogging tests were conducted along a $12 \mathrm{~m}$ walkway with a middle section of $7 \mathrm{~m}$ designated for data collection. The PEDAR insole system (Novel, GmBH, Munich, Germany) was used to measure the pressure and force between the plantar surface of the foot and the shoe. The PEDAR insole is a pressure distribution measuring device based on the capacitance principle. The insoles are approximately $2.6 \mathrm{~mm}$ thick and contain 99 sensors. The insole system is able to measure pressures up to $120 \mathrm{~N} / \mathrm{cm}^{2}$.

Three types of women's fashion shoes (sized 37 EUR) with different net heel height (flat: $0.8 \mathrm{~cm}$, low: $4.0 \mathrm{~cm}$, and high: $6.6 \mathrm{~cm}$ ), according to difference between the back and forefoot part of shoe (Fig. 1). All the shoes were commercially available with similar design, construction, and material. In addition, these footwear selected were also similar in terms of forefoot width and straps, as these may potentially affects the results.

\subsection{Procedures}

Preliminary trials showed that all subjects have a heel-toe running pattern, and they were asked to perform jogging at their own natural pace for several trials with different testing shoes, the running speed was found to be between $2 \mathrm{~m} / \mathrm{s}$ to $3 \mathrm{~m} / \mathrm{s}$. This procedure ensures that the subject become accustomed to the testing insoles. During the test with full scale data collection, participants were asked to jog three times at her natural speed in each study condition. Furthermore, there was a six-minutes resting interval between tests of different shoe conditions in order to prevent any fatigue effect, and resting time was confirmed by the subjects during preliminary trials for this short jogging distance. The three conditions of different heel height were tested in a randomized sequence.

\subsection{Data analysis}

Data analysis was based tests from the left feet of all participants. The foot was divided into eight regions of masks (Fig. 1): big toe (BT), lesser toes (LT), medial forefoot (M1, nearly the first metatarsal), 

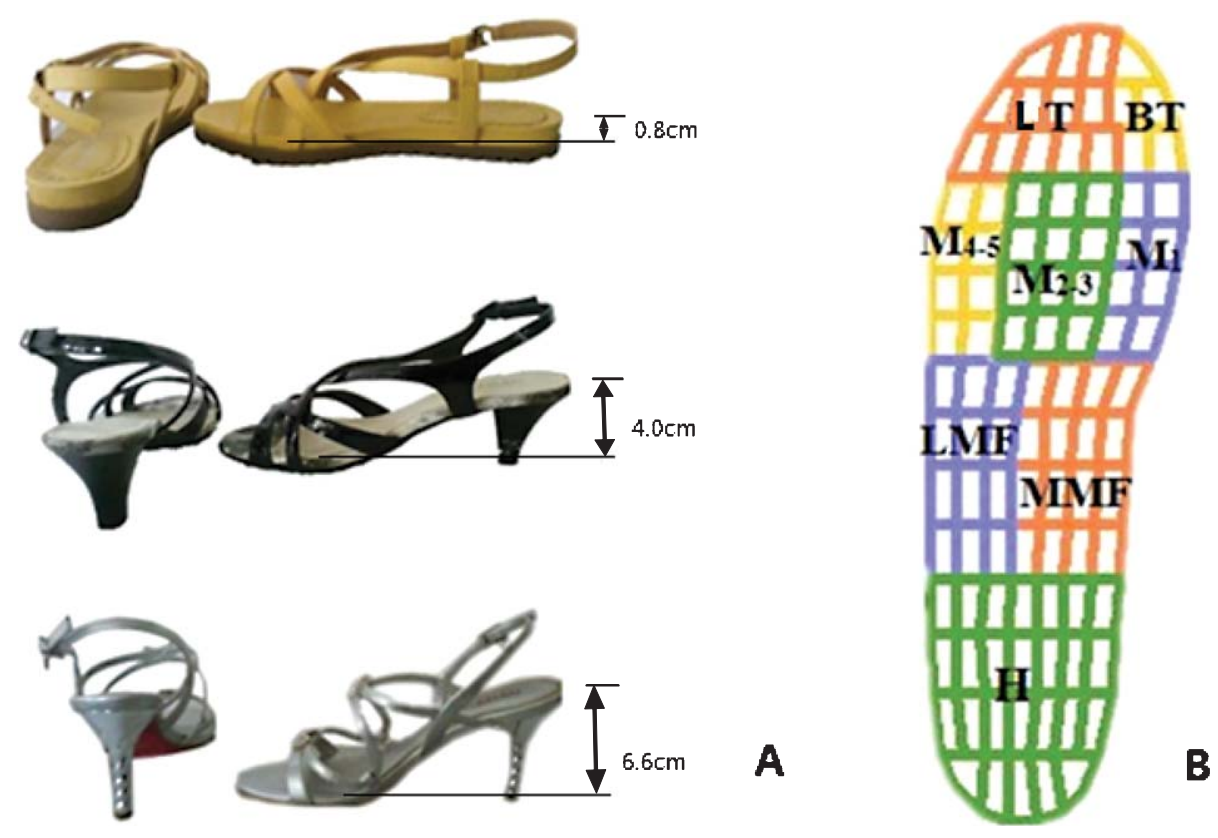

Fig. 1. A: Shoes with different heel height; B: The mask definition of the plantar area.

middle forefoot (M2-3, nearly the second and third metatarsal), lateral forefoot (M4-5, nearly the fourth and fifth metatarsal), medial midfoot (MMF), lateral midfoot (LMF) and heel (H). For each of these regions the following parameters were assessed: contact area (CA) in $\mathrm{cm}^{2}$, maximum force (MF) in $\mathrm{N}$, peak pressure (PP) in $\mathrm{N} / \mathrm{cm}^{2}$, force time integral (FTI) in Ns. The FTI provides an understanding of the load distribution applied over time.

SPSS 17.0 for Windows was used in the statistical analysis. Data from clinical evaluation and physical measurements were input to the SPSS program. Analysis of variance (ANOVA) was employed to study the effects of heel height, and Tukey test was used for post hoc comparison with the level of statistical significance set at a level of $5 \%$.

\section{Results}

The data of the key foot areas is shown in Fig. 2. It is clearly shown that jogging with high heeled shoes has significantly increased the maximum force in BT and M1 regions $(p<0.05)$. However, less force was found to be acting on the LT, M4-5, MMF, LMF and H regions when comparing the data of the high heeled shoes to low and flat heeled shoes jogging $(p<0.05)$. It is also evident that the M2-3 region had no significant alteration in maximum force. As shown in Fig. 3, the peak pressure exhibited patterns similar to that of maximum force (Fig. 3), i.e., lower peak pressure in M4-5, MMF, and LMF regions and higher peak pressure in BT and M1 regions $(p<0.05)$. A sharp decrease of about $70 \%$ in peak pressure was observed in the midfoot and M4-5 region when comparing high heeled shoes data to the low and flat heeled shoes data. However, the increase in BT and M1 was only about $20 \%$ with the high heeled shoes jogging. A similar pattern was observed in the data of force-time integral (Fig. 4). The differences were especially accentuated on the midfoot and BT region with a decrease of about $90 \%$ and an increase of $70 \%$, respectively.

As shown in Fig. 5, the contact area was smaller in the M4-5 region and midfoot for the high heeled jogging compared with the flat heeled jogging $(p<0.05)$. Particularly, it almost reduced to zero in the LMF region. Meanwhile, the contact area was lager in the OT and M1 region with high heels $(p<0.05)$.

\section{Discussion}

The results in present study suggests that increasing heel height would change maximum force, peak 


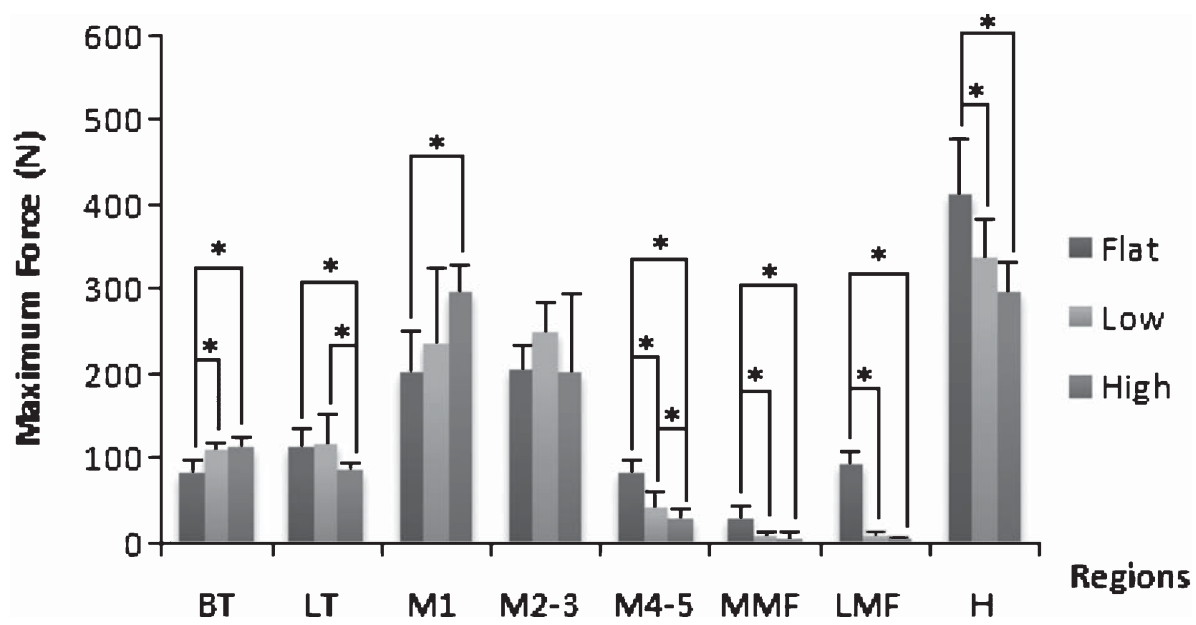

Fig. 2. Maximum force during jogging with different heel heights. *indicates significant difference $(p<0.05)$.

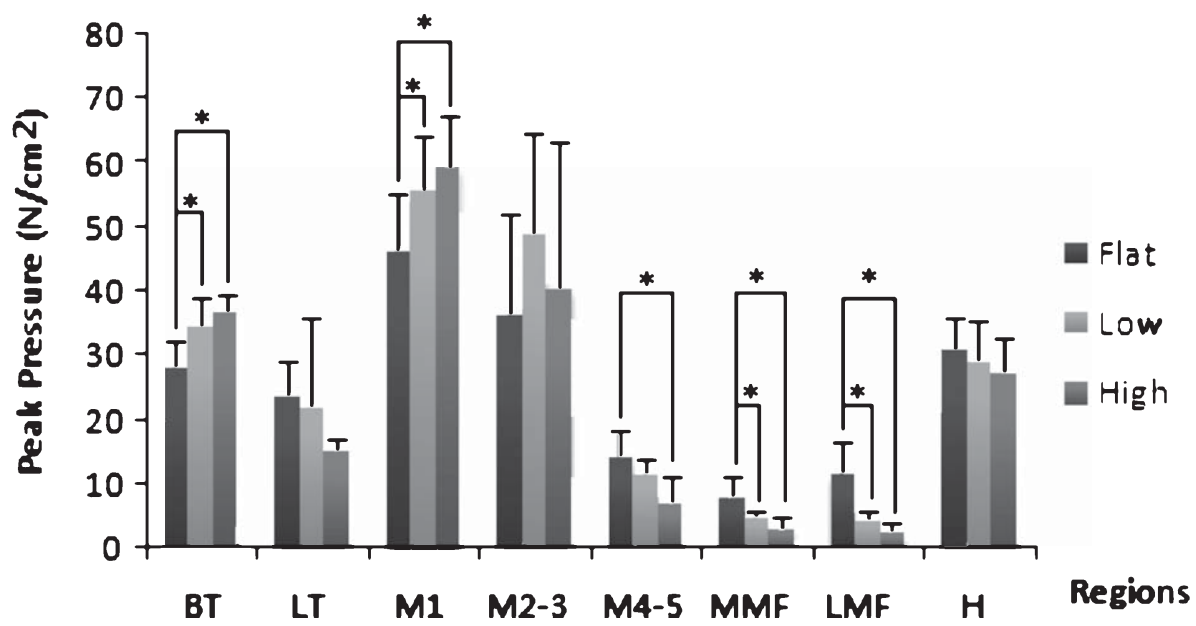

Fig. 3. Peak pressure during jogging with different heel heights. ${ }^{*}$ indicates significant difference $(p<0.05)$.

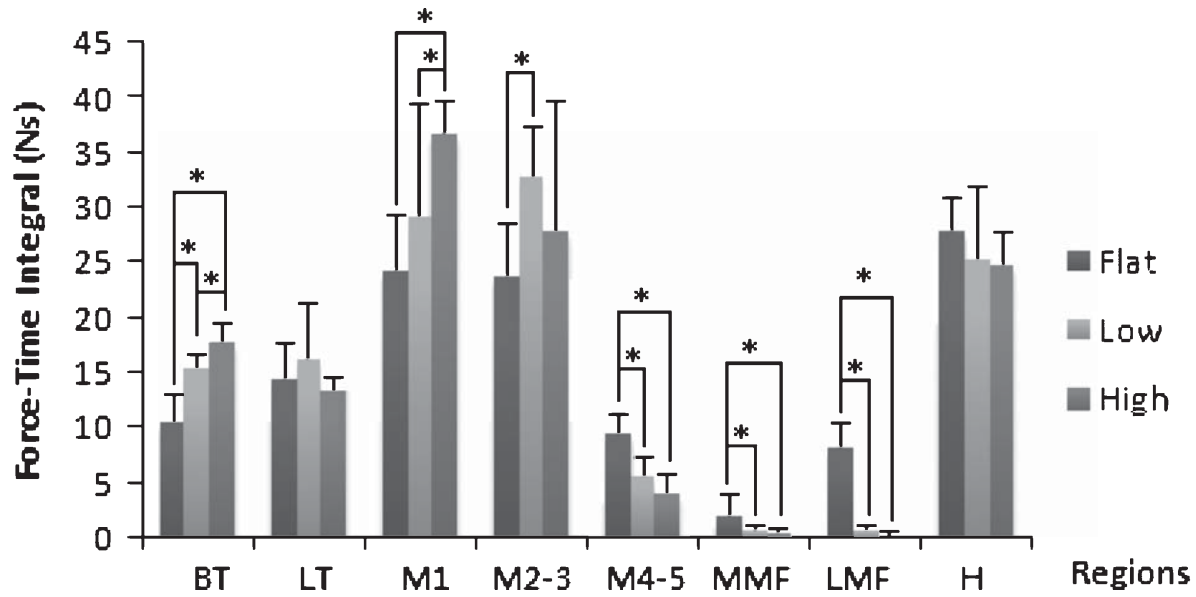

Fig. 4. Force-time integral during jogging with different heel heights. ${ }^{*}$ indicates significant difference $(p<0.05)$. 


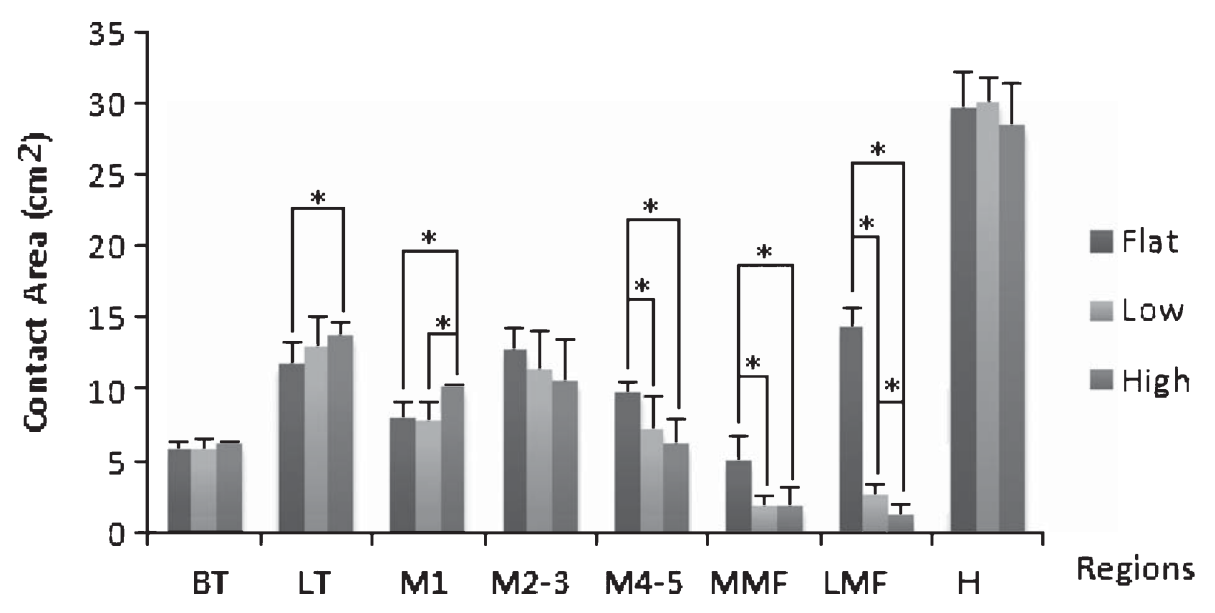

Fig. 5. Contact area during jogging with different heel heights. ${ }^{*}$ indicates significant difference $(p<0.05)$.

pressure, force-time integral, and contact area under the forefoot surface (BT, OT, M1, M2-3 and M4-5 regions) during jogging. All the measured parameters except the contact area value were increased in the medial forefoot but decreased in the lateral forefoot. In other words, the high pressure zones shifted from the lateral forefoot to the medial forefoot. This trend was consistent with those of previous studies $[11,13$, $15,16]$. Interestingly, there was a distinctive difference between the data for the M2-3 regions of this work and previous researches on high heeled walking, in which no significant difference was observed in the M2-3 regions. This difference could be due to the fact that the plantar pressure focused on the medial forefoot much more during jogging with higher values than walking [23]. The alterations of the pressure distibution might lead to the discomfort of the foot and may cause some foot injuries such as plantar fasciitis [24]. Because high forefoot loading means the posture of ankle was positioned in plantar flexed, which would shrink calf muscles [29]. In this case, changing high heel to flat-heeled shoes can cause excessive load on Achilles tendon and plantar fascia.

Under the midfoot area, there is a clear decreasing trend of loading with increasing heel height. This maybe caused by a decreased roll forward over the midfoot in high-heeled shoes [25]. These findings were in a reasonable agreement with the study of Lee et al. [22], but the values of peak pressure were different from a previous study which reported that the midfoot pressure increased after the heel height was increased [12]. Under the condition of the experiments of this work, contact area of the midfoot was very limited when wearing high-heeled shoes, which may have contributed to significant reduction of peak loading in this region. The decrease of midfoot support function may lead to high loading on the knee joint [26]. Another aspect requires attention is that most high-heel shoe's sole material was very rigid, so both structure and material could severely restrict the contact area of the midfoot. This is the reason why the contact area could be effectively expanded when using some arch support insert over the midfoot section [22].

Previous work on walking suggested that there is a significant load decrease in the heel region during gait in high-heeled shoes [12-14]. In high heel jogging movement, heel region was shown a similar trend. The findings reported in this work confirm that wearing high-heeled shoes significantly alters the normal function of the foot. Because of this complication, compensations must occur at the knee, hip even the back of the subject to maintain stability and progression during walking or jogging [9, 27]. As highlighted in the introduction section, in a modern life, in some cases, the high heel wearers have to move in relative high speed similar to the pace of jogging. The data showed a clear difference between walking data and higher intensity jogging data, which is important in improving the understanding of jogging with high heels and injury prevention. It can also potentially guide the development of adaptive exercise schemes in between daily activities with high heels. However, there are several limitations of our study that should be noted. To reflect the realistic conditions, the data was based natural jogging speed rather than controlled speed. This may bring variation between 
subjects. In addition, the subjects selected in this testing all were experienced high heel shoes user. People who are unaccustomed to high heel shoes may have a different loading pattern thus different biomechanical response. Only ten subjects were selected in this test, further study shall recruit more participants. The jogging speed was only generally screened during the experiment, further study should also control the speed under more specific level. The implication of the data on potential design improvement in safety improvement will be addressed in future works. The difference between jogging and walking suggests that the weakening of midfoot support function during high heel jogging shall be concerned; factors that raise the perception of this region may have substantial influence on high heel jogging safety.

\section{Conclusions}

In summary, the results of the current study suggest that jogging with high heel shoes predisposes an individual to forefoot pain. This was reflected by significant increases in the loading parameters compared to that of wearing flat shoes. The work revealed the different effects of heel height on the plantar pressure between walking and jogging with high heeled shoes. The data showed that the midfoot was nearly unsupported region during high-heeled jogging, high heel shoes enforce foot into a position in which these forces could have a deleterious effect. One potential approach to improve the performance of high heel shoes in new footwear design could potentially be achieved through improving sustainable function of midfoot.

\section{Acknowledgements}

The study sponsored by National Natural Science Foundation of China (81301600), K.C.Wong Magna Fund in Ningbo University, and Ningbo Natural Science Foundation (2013A610262).

\section{References}

[1] C. Frey, F. Thompson and J. Smith, et al., American Orthopaedic Foot and Ankle Society women's shoe survey, Foot and Ankle 14 (1993), 78-81.

[2] M. Esenyel, K. Walsh and J.G. Walden, et al., Kinetics of high-heeled gait, Journal of the American Podiatric Medical Association 93 (2003), 27-32.
[3] M.G. Mandato and E. Nester, The effects of increasing heel height on forefoot peak pressure, J Am Podiatr Med Assoc 89 (1999), 75-80

[4] J.P. Corrigan, D.P. Moore and M. Stevens, Effect of heel height on forefoot loading, Foot Ankle 14 (1993), 148-152.

[5] N.L. Broch, T. Wyller and H. Steen, Effects of heel height and shoe shape on the compressive load between foot and base: A graphic analysis of principle. J Am Podiatr Med Assoc 94 (2004), 461-469.

[6] I.D. McBride, U.P. Wyss and T.D. Cooke, et al., First metatarsophalangeal joint reaction forces during high-heel gait, Foot Ankle 11 (1991), 282-288.

[7] J. Dawson, M. Thorogood and S.A. Marks, et al., The prevalence of foot problems in older women: A cause for concern, J Public Health Med 24 (2002), 77-84.

[8] J. Dawson, E. Juszczak and M. Thorogood, et al., An investigation of risk factors for symptomatic osteoarthritis of the knee in women using a life course approach, $J$ Epidemiol Community Health 57 (2003), 823-830.

[9] D.C. Kerrigan, M.K. Todd and P.O. Riley, Knee osteoarthritis and high-heeled shoes, Lancet 351 (1998), 1399-1401.

[10] R.E. Snow and K.R. Williams, High heeled shoes: Their effect on center of mass position, posture, three-dimensional kinematics, rearfoot motion, and ground reaction forces, Arch Phys Med Rehabil 75 (1994), 568-576.

[11] J. Yu, J.T.M. Cheung and Y. Fan, et al., Development of a finite element model of female foot for high-heeled shoe design, Clinical Biomechanics 23 (2008), 31-38.

[12] Y. Luximon, A. Luximon and J. Yu, et al., Biomechanical evaluation of heel elevation on load transfer - experimental measurement and finite element analysis, Acta Mech Sin 28 (2012), 232-240.

[13] C.M. Speksnijder, R. Munckho and S. Moonen, et al., The higher the heel the forefoot-pressure in ten healthy women, The Foot 15 (2005), 17-21.

[14] M. Cernekova and P. Hlavacek, The influence of heel height on plantar pressure, Clinical Biomechanics 23 (2008), 667-668.

[15] Y. Cong, J.T.M. Cheung and A. Leung, et al., Effect of heel height on in-shoe localized triaxial stresses, Journal of Biomechanics 44 (2011), 2267-2272.

[16] Y.H. Lee, W.H. Hong and H.C., Chen, et al. Influence of heel height and shoe insert on comfort perception and biomechanical performance of young female adults during walking, Foot \& Ankle International 26 (2005), 1042-1048.

[17] Y.D. Gu, M. Rong, G.Q. and Ruan, The outsole pressure distribution character during high-heeled walking, Procedia Environmental Sciences 8 (2011), 464-468.

[18] D.E. Lieberman, M. Venkadesan and W.A. Werbel, et al., Foot strike patterns and collision forces in habitually barefoot versus shod runners, Nature 463 (2010), 531-535.

[19] F. Fourchet, L. Kelly and C. Horobeanu, et al., Comparison of plantar pressure distribution in adolescent runners at low vs. high running velocity, Gait \& Posture 35 (2012), 685-687.

[20] B. Chyckpaiwong, J.A. Nunley and N.A. Mall, et al., The effect of foot type on in-shoe plantar pressure during walking and running, Gait \& Posture 28 (2008), 405-411.

[21] T.S. Keller and Weisberger, et al., Relationship between vertical ground reaction force and speed during walking, slow jogging, and running, Clinical Biomechanics 11 (1996), 253-259.

[22] Y.H. Lee and W.H. Hong, Effects of shoe inserts and heel height on foot pressure, impact force, and perceived comfort during walking, Applied Ergonomics 36 (2005), 335-362. 
[23] L.Y. Guo, C.F. Lin and C.H. Yang, et al. Effect on plantar pressure distribution with wearing different base size of high-heel shoes during walking and slow running, Journal of Mechanics in Medicine and Biology 12 (2012), 1250018-1-1250018-11.

[24] E. Morag and P.R. Cavanagh, Structural and functional predictors of regional peak pressures under the foot during walking, J Biomech 32 (1999), 359-370.

[25] I.D. McBride, U.P. Wyss and T. Cooke, et al., First metatarsophalangeal joint reaction forces during high-heel gait, Foot Ankle 11 (1991), 282-288.

[26] D.C. Kerrigan, J.L. Johansson and M.G. Bryant, et al. Moderate-heeled shoes and knee joint torques relevant to the development and progression of knee osteoarthritis, Arch Phys Med Rehabil 86 (2005), 871-875.

[27] R. Iqbal, W. Mishra, S. Maulik and A.M. Chandra, Study on lumbar kinematics and the risk of low back disorder in female university students by using shoes of different heel heights, Work 41 (2012), 2521-2526.

[28] M. Linder and C.L. Saltzman, A history of medical scientists on high heels, Int J Health Serv 28 (1998), 201-225.

[29] A. Gefen, M. Megido-Ravid, Y. Itzchak and M. Arcan, Analysis of muscular fatigue and foot stability during high-heeled gait, Gait Posture 15 (2002), 56-63. 

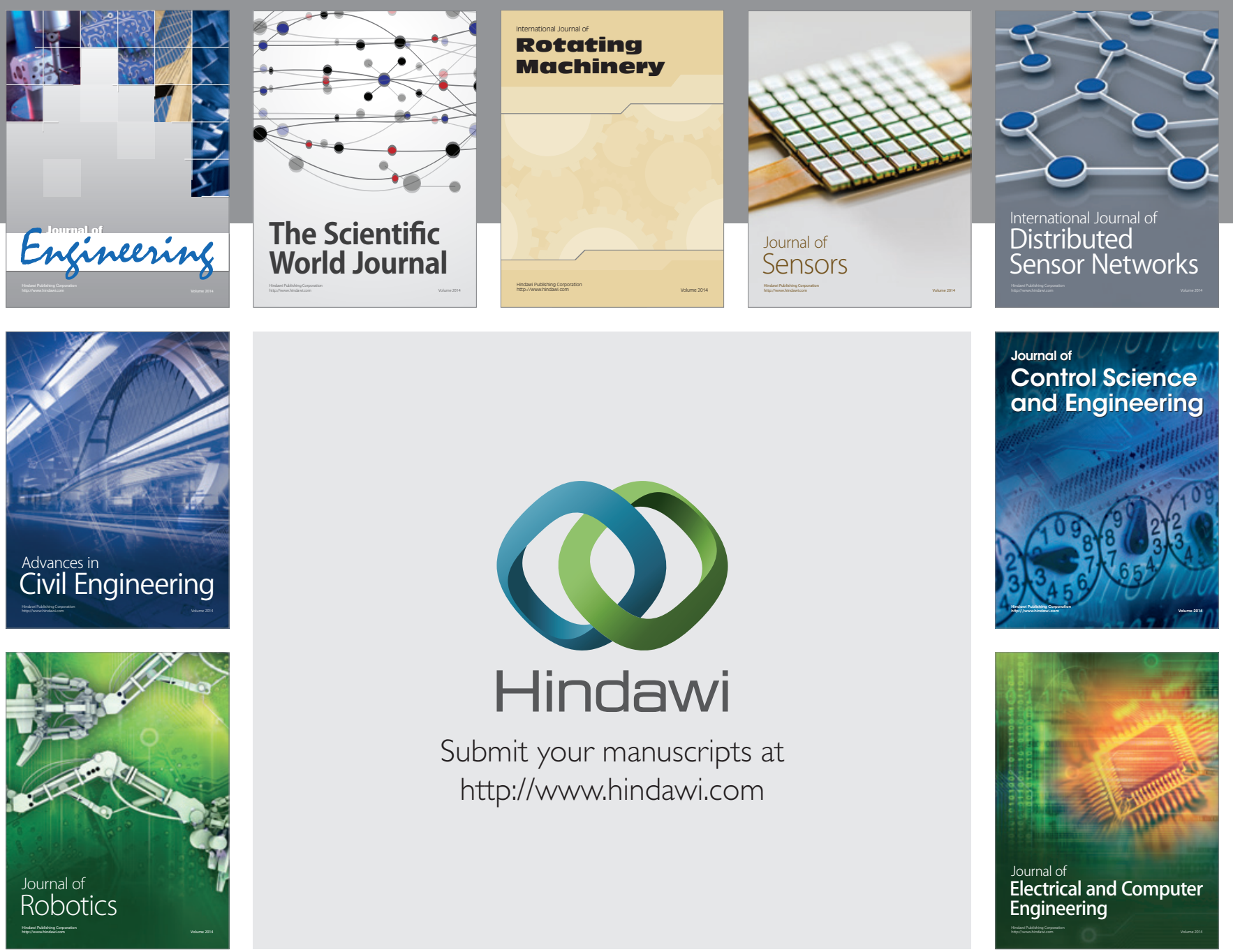

Submit your manuscripts at

http://www.hindawi.com
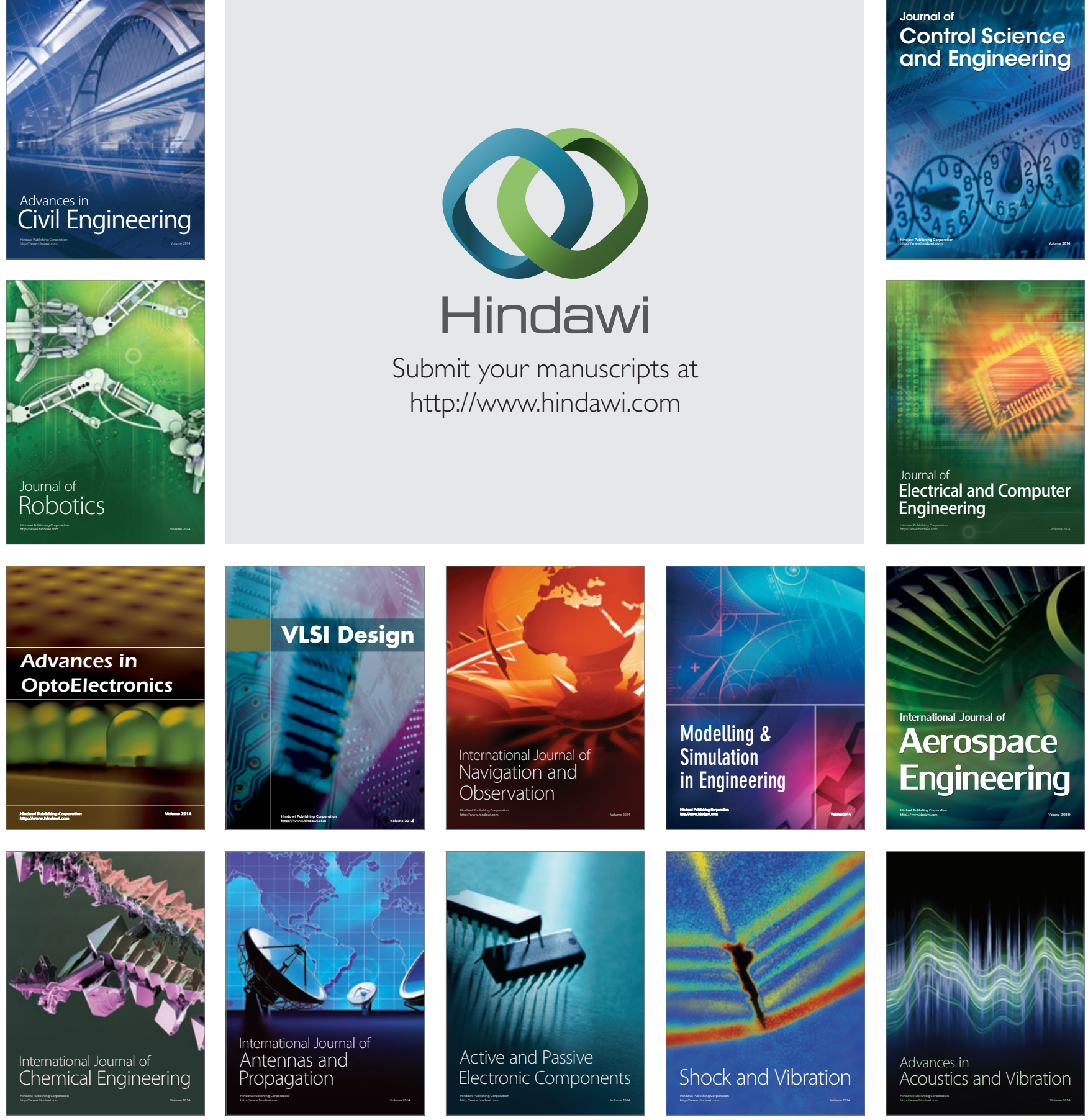